Health Place. 2014 July ; 28: 177-186. doi:10.1016/j.healthplace.2014.05.003.

\title{
Multilevel examination of the association of urbanization with inflammation in Chinese adults
}

\author{
Amanda L. Thompson ${ }^{1,2}$, Kelly M. Houck ${ }^{1,2,3}$, Linda Adair ${ }^{2,3}$, Penny Gordon-Larsen ${ }^{2,3}$, and \\ Barry Popkin ${ }^{2,3}$ \\ ${ }^{1}$ Department of Anthropology, University of North Carolina at Chapel Hill, Chapel Hill, NC 27599, \\ USA \\ ${ }^{2}$ Carolina Population Center, University of North Carolina at Chapel Hill, 123 W Franklin St, \\ Chapel Hill, NC, 27516, USA \\ ${ }^{3}$ Department of Nutrition, Gillings School of Global Public Health, University of North Carolina at \\ Chapel Hill, Chapel Hill, NC, 27599, USA
}

\begin{abstract}
We examine the associations between overall urbanicity and specific physical and social components of community-level urbanization with C-reactive protein (CRP) in adults participating in the China Health and Nutrition Study. Higher overall urbanicity and environment-related urbanicity component scores, including education, housing quality, and access to markets, were associated with elevated CRP in multilevel models controlling for clustering by community. These associations differed by age and gender and persisted after controlling for individual-level anthropometric, diet, and pathogenic risk factors. These results highlight the importance of place in relation to inflammation across the spectrum of rural and urban environments.
\end{abstract}

\section{Keywords}

China; C-reactive protein; Inflammation; Urbanization; Neighborhood health

\section{Introduction}

Urbanization, the process of increasing urban size, density and heterogeneity (Vlahov and Galea, 2002), is widely recognized as a driver of globally changing patterns of health and disease. Worldwide, the prevalence of obesity and chronic diseases, such as hypertension

\footnotetext{
C 2014 Elsevier Ltd. All rights reserved

Corresponding author: Amanda L. Thompson 123 W. Franklin St., CB \#8120 Chapel Hill, NC 27516 919-843-6255 (phone) 919-966-6638 (fax) althomps@email.unc.edu.

Co-author e-mails: Kelly M. Houck: khouck@live.unc.edu

Linda Adair: linda_adair@unc.edu

Penny Gordon-Larsen: pglarsen@unc.edu

Barry Popkin: popkin@unc.edu

Publisher's Disclaimer: This is a PDF file of an unedited manuscript that has been accepted for publication. As a service to our customers we are providing this early version of the manuscript. The manuscript will undergo copyediting, typesetting, and review of the resulting proof before it is published in its final citable form. Please note that during the production process errors may be discovered which could affect the content, and all legal disclaimers that apply to the journal pertain.
} 
and diabetes, is significantly higher in urban than in rural areas (Popkin et al., 2012), leading some researchers to characterize urbanization as a process creating an "urban health penalty" (Vlahov et al., 2005). The pathways linking urbanization and health, however, are multifaceted. Urbanization offers opportunities for improvements in health through increased access to health care, economic opportunities, and improved basic infrastructure, but also substantial health risks from pollution, occupational and traffic hazards, Westernized diets, and reduced physical activity (Gong et al., 2012; Ng et al., 2009; Popkin 1999; Popkin and Du, 2003; Vlahov et al., 2005). How these factors independently and jointly influence individuals' susceptibility to disease is not well characterized. Parallel research in neighborhoods and health documents the positive and negative role of place on health outcomes through a number of interrelated and mediating pathways (Diez Roux and Mair, 2010). Contextual attributes of place (availability of grocery stores, housing quality and neighborhood safety), health behavior (physical activity and alcohol use), disease exposures, and psychosocial factors (anxiety, stress, and depression) have all been proposed to link local environments to health outcomes (Daniel et al., 2008). These contributors to the effects of neighborhood on health are likely to overlap those linking urbanization to health and to share similar behavioral and biological pathways.

Inflammation, induced by immune recognition of infection or tissue damage, is one such pathway that may link physical and social environments to poor health outcomes, such as cardiovascular disease (Pearson et al., 2004), diabetes (Pradhan et al., 2001) and depression (Hansel et al., 2010). C-reactive protein (CRP), a commonly measured inflammatory marker, responds to the pathogen exposures (Kushner et al., 2006; McDade et al., 2008), adiposity (Kushner et al., 2006; McDade et al., 2008) and psychosocial stress (Browning et al., 2012; Hansel et al., 2010) that characterize neighborhoods and accompany urbanization. Adult CRP levels are significantly associated with individual and neighborhood socioeconomic status (Gallo et al., 2012; Holmes and Marcelli, 2012; Petersen et al., 2008; Pollitt et al., 2007), neighborhood deprivation, safety, and cohesion (Browning et al., 2012; Nazmi et al., 2010a) and housing conditions (Cagney and Cornwell, 2010; Williams and McDade, 2009). However, much of this research on neighborhood characteristics and inflammation has focused on the United States and other Western countries. The impact of pathogenic, obesogenic, and psychosocial neighborhood characteristics in populations undergoing rapid development and urbanization remains less well characterized (Wen et al., 2010).

Previous studies in developing countries have shown that CRP levels differ across rural and urban populations (Yajnik et al., 2008; Ye et al., 2007), but this research has tended to focus on urban-rural comparisons or on specific urban populations. An urban/rural dichotomy does not adequately distinguish living conditions experienced at different stages of urbanization (Dahly and Adair, 2007; Jones-Smith and Popkin, 2010; McDade and Adair, 2001), particularly in contexts of rapid social and environmental change. Health effects are likely to differ across the spectrum of urbanization (Gong et al., 2012; Li et al., 2012). We capitalize on the rich community, household, and individual-level data collected by the China Health and Nutrition survey across a range of urban and rural communities in nine provinces in eastern China to test the association between urbanization and inflammation using a previously validated urbanicity scale (Jones-Smith and Popkin, 2010). This scale, 
comprised of 12 diverse components ranging from economic factors to urban infrastructure and healthcare, provides a comprehensive measure of urbanization (Jones-Smith and Popkin, 2010).

China is an ideal setting to test how community-level urbanization relates to inflammation, having witnessed unprecedented rates of urbanization and economic growth (Gong et al., 2012) accompanied by dramatic changes in landscapes (Li et al., 2012) and lifestyles (Popkin and Du, 2003). Patterns and rates of change have not been universal. Some rural areas have seen rapid population growth and changes within specific dimensions, such as access to modern markets, while some cities have lost services and physical infrastructure with economic development ( $\mathrm{Li}$ et al., 2012). Given this heterogeneity, understanding the overall impact of urbanization and the elements of the urban environment, such as sanitation infrastructure or access to markets, most associated with inflammation is important for identifying the pathways placing individuals at risk and for informing community-level prevention efforts. Consequently, we examined both the overall score from the multicomponent urbanicity scale and the 12 scale components using multilevel models controlling for clustering by community. We hypothesized that: 1) overall urbanicity score, as a comprehensive measure of urbanization, would be associated with an increased risk of moderately-elevated CRP and 2) components associated with pathogen exposure (e.g. sanitation and housing quality), adiposity (e.g. access to markets and transportation infrastructure), and psychosocial stress (e.g. population density and income diversity) would be individually associated with moderately-elevated CRP. Finally to more clearly examine the potential pathways linking urbanization to chronic disease risk, we tested whether these community-level components had persistent associations with CRP after controlling for a host of individual-level sociodemographic, pathogenic, and obesogenic risk factors.

\section{Sample and Methods}

\section{The China Health and Nutrition Survey (CHNS)}

The CHNS collected health, nutrition, and economic data from 228 communities across nine diverse provinces (Guangxi, Guizhou, Heilongjiang, Henan, Hubei, Hunan, Jiangsu, Liaoning, and Shandong). The CHNS was designed to provide representation of rural, urban, and suburban areas varying substantially in geography, economic development, public resources, and health indicators with a focus on examining diet, physical activity, health and behavior changes in the context of urbanization and economic change (Popkin et al., 2010). Multistage, random-cluster sampling and weighted sampling were used to select participating communities. Using this sampling strategy, two cities (one large, usually the provincial capital, and one small city, usually a lower income one) and four counties (stratified by income: one high, one low, and two middle income counties) were selected per province. Within cities, two urban and two suburban communities were randomly selected; within counties, one community in the capital city and three rural villages were randomly chosen. In each community, 20 households were randomly selected and all household members were interviewed. The 2009 wave consists of 216 communities and includes 36 urban neighborhoods, 36 suburban neighborhoods, 36 towns and 108 villages. The CHNS cohort initially mirrored national age-gender-education profiles (Wang, 2002) and by 2011, 
the provinces included in the CHNS sample represented $47 \%$ of China's population (according to 2010 census).

Survey procedures have been described in detail (Popkin et al., 2010). Briefly, eight rounds of surveys were conducted at the individual, household, and community-levels from 19892009 and fasting blood was first collected in the 2009 survey. All measures for the current study come from the 2009 wave of data collection. The analytic sample for the current analyses includes men $(n=3861)$ and non-pregnant women $(n=4388)$ over the age of 18 with measured hs-CRP at the 2009 exam. Subjects were excluded if they were pregnant $(n=58)$ or had hs-CRP >10mg/L ( $\mathrm{n}=309$ ), a commonly used indicator of current infection (Pearson et al., 2004). This study protocol and analysis was approved by the Institutional Review Board at the University of North Carolina at Chapel Hill, the China-Japan Friendship Hospital, Ministry of Health, and Institute of Nutrition and Food Safety, China Centers for Disease Control. Subjects gave informed consent for participation.

\section{Measures}

\section{High-Sensitivity C-Reactive Protein (hs-CRP)}

Blood $(12 \mathrm{~mL})$ was collected by venipuncture after an overnight fast. Whole blood was immediately centrifuged and plasma and serum samples were frozen and stored for analysis. Hs-CRP was measured using the immunoturbidmetric method (Hitachi 7600 automated analyzer) with Denka (Seiken, Japan) reagents. This assay has a range of $0.1-320 \mathrm{mg} / \mathrm{L}$ and a coefficient of variation $<7.0 \%$ across assays. All samples were processed in a national lab in Beijing, awarded a medical laboratory accreditation certificate (ISO 15189:2007). Moderate elevations in CRP were defined as values between 3-10mg/L and were compared to a reference group with hs-CRP $<3 \mathrm{mg} / \mathrm{L}$, since values above this $3 \mathrm{mg} / \mathrm{L}$ cut-point are associated with high risk of future cardiovascular events (Pearson et al., 2004)

\section{Urbanicity Scale}

Urbanicity was defined using a 12-component index capturing community-level physical, social, cultural, and economic environments designed and validated for the CHNS (JonesSmith and Popkin, 2010). This scale represents a broad-based measure of the elements of modernization that have potential health effects. Components (Table 1) capture heterogeneity in presence/absence or number of facilities within the community, facility characteristics, access to media or infrastructure, and the average proportion of individuals and households having a specific education or income level. The variables measuring the proportion of households were obtained from the CHNS household responses and the remaining variables were derived from the CHNS community-level survey provided to community officials. As described by Jones-Smith and Popkin (2010), scoring distributions were variable across components so the median value component value was set as half the total points and each of the components were scaled to 0-10. Each component was then weighted equally in the overall index and added together for an overall maximum possible score of 120 . This scale has been validated for content validity, reliability $(a=0.85-0.89$ across all study years), and stability across study years ( $\mathrm{r}=0.90-0.94)$. 
We first examine whether total urbanicity score is associated with the likelihood of moderate inflammation. Based on the non-linearity of the association between urbanization and inflammation, we categorized the total urbanicity scale scores (range: 30.4-106.6) into tertiles representing low (<59.0), medium (59.0-82.2) and high (>82.3) urbanization levels. In a second set of analyses, we explore how each specific component of the scale relates to the risk of elevated CRP since China's rapid urbanization has been characterized by considerable spatial and temporal variability in environmental and social changes. Scores for each component were standardized to facilitate comparison between components and the presented odds ratios represent a 1 -standard deviation change in the urbanicity component score.

\section{Anthropometry}

Two trained health workers collected weight, height, and waist circumference following standard protocols. Weight was measured without shoes and in light clothing to the nearest $0.1 \mathrm{~kg}$ on a calibrated beam scale. Height was measured without shoes to the nearest $0.2 \mathrm{~cm}$ using a portable stadiometer and averaged across all repeated measures for analyses. BMI was calculated as $\mathrm{kg} / \mathrm{m}^{2}$. Overweight and obesity were defined using the China-specific cutpoints of $24 \mathrm{~kg} / \mathrm{m}^{2}$ and $28 \mathrm{~kg} / \mathrm{m}^{2}$, respectively (Working Group on Obesity in China, 2004). Waist circumference was measured midway between the lowest rib and the iliac crest using a non-elastic tape. High waist circumference was defined using Asia-specific cut-points of $>80 \mathrm{~cm}$ for women and $>90 \mathrm{~cm}$ for men (Alberti et al., 2006).

\section{Covariates}

Additional demographic variables that could influence the association between communitylevel measures of urbanicity and inflammation-- income, education, physical activity level, high fat diet, illness history, and current smoking-- were included in analysis. Income was derived from total household income and measured in yuan. Education was defined as the highest current level at the time of the survey, categorized into completion of less than primary, primary, secondary, and post-secondary education. Physical activity was reported using a detailed 7-day physical activity recall instrument. Leisure activities were assigned Metabolic Equivalent (MET) values using the Compendium of Physical Activity (Ainsworth et al., 2000), previously validated for use in China ( $\mathrm{Ng}$ et al., 2009) and dichotomized into active (vs. non-active). Active was defined as $\geq 27$ METs per week of leisure activity, equivalent to $\geq 1$ hour per week of moderate-intensity exercise such as brisk walking. Dietary fat intake was derived from individual-level 24-hour recalls taken on three consecutive days. Macronutrient values were estimated from the 2002/2004 Chinese Food Composition Table and diets were considered high fat if $30 \%$ or more of the total daily energy intake was derived from fat (Popkin et al., 2002). Participants' self-reported infectious illness history over the 4 weeks preceding the survey was included in the model as a dichotomous variable (any symptoms vs. no symptoms) since differences in pathogen exposure could mediate the association between community-level urbanicity and individual inflammation. Smoking was included as a dichotomous variable based on whether participants were currently smoking at the 2009 survey. Region (North vs. South) was included as a covariate due differences in health indicators and longevity by region in China (Chen et al., 2013). 


\section{Statistical methods}

We tested for differences in individual-level variables across low, medium, and high urbanicity separately for men and women using ANOVA tests for continuous outcomes and chi-square tests for categorical outcomes. Multilevel logistic models controlling for the fixed effect of age and the random effect of community were used to test for associations between measures of urbanicity and its components and moderate inflammation. Intraclass correlation coefficients of CRP within communities were estimated using the mixed model variance components. We tested whether the odds of elevated CRP differed across low, medium, and high urbanicity controlling for the fixed effects of age and income and the random effects of community. Initial model specification identified significant effect modification by sex and all models were subsequently stratified by sex. Similarly, there was evidence for significant effect modification by age and an interaction term between age strata and urbanicity was included in the sex-stratified models. Next, we examined whether this association between urbanization and elevated CRP differed across the components of urbanization, to identify what aspects of urbanization place individuals at risk for inflammation. The 12 urbanicity scale components were tested in separate models controlling for the fixed effect of age and random effect of community, stratified by sex, with interaction terms between the components and age strata. In the final set of models, previously identified individual-level risk factors for elevated CRP, overweight, high waist circumference, high fat diets, low physical activity, infectious illness symptoms, smoking, income, and education (Kushner et al., 2006), were added to these component models in groups (sociodemographic, obesogenic, and pathogenic) to test whether their inclusion attenuated the association between urbanization components and elevated CRP. These models control for the fixed effects of the previously mentioned individual-level risk factors, region and the random effect of community. All models were specified to provide an estimate of the slope with urbanicity for each age strata (18-39.99, 40-60, >60 years). We calculated and plotted the predicted probability of moderate inflammation with increasing urbanicity for each of the age strata in both the minimally and fully-adjusted models to more clearly illustrate model results.

All analyses were conducted in Stata version 12 (College Park, Texas).

\section{Results}

\section{Sample Descriptives}

Mean CRP levels increased significantly with age in both men and women. Younger men (aged 18-40 years) had higher CRP levels than younger women $(1.39 \pm 1.67 \mathrm{mg} / \mathrm{L} v s$. $1.08 \pm 1.62 \mathrm{mg} / \mathrm{L}, \mathrm{p}<0.001$, respectively). Conversely, older men ( $>60$ years) had lower mean CRP levels than older women $(2.05 \pm 2.05 \mathrm{mg} / \mathrm{L} v s .2 .27 \pm 2.14 \mathrm{mg} / \mathrm{L}, \mathrm{p}=0.01$, respectively $)$. There were no significant differences between middle-aged men and women $(1.72 \pm 1.91$ $\mathrm{mg} / \mathrm{L}$ vs $1.63 \pm 1.92 \mathrm{mg} / \mathrm{L}, \mathrm{p}=0.16$ ).

Individual-level risk factors previously shown to be associated with moderate elevations of CRP --overweight, obesity, and high waist circumference-- significantly increased across urbanicity tertiles in men but not women (Table 2). In both sexes, household income, highest 
level of education completed, and participation in leisure time physical activity were higher and the prevalence of smoking lower with greater urbanicity. The percent of variance in CRP between communities, estimated from the multilevel model without covariates, was $1.4 \%$ for men and $3.0 \%$ for women.

\section{Urbanicity and CRP}

Increasing urbanicity was associated with a greater probability of elevated CRP in both men and women, but the effects differed across age strata (Figure 1). Younger men (18-40) were significantly more likely to have higher odds of moderate inflammation at middle and high levels of urbanicity (OR: 1.69, 95\% CI: 1.08-2.64 and OR: 1.78, 95\% CI: 1.14-2.77, respectively) than at low urbanicity. The pattern was less consistent and not statistically significant at older ages. Conversely, overall urbanicity score was associated with the odds of moderate inflammation in women over 40. Women aged 40-60 had higher odds of elevated CRP at high urbanicity (OR: 1.38, 95\%CI: 1.04-1.83) while women over 60 had higher odds of elevated CRP at middle (OR 1.47, 95\% CI 1.05-2.07) and high (OR 1.43, 95\% CI (1.04-1.97) urbanicity.

\section{Urbanicity Components and CRP}

As with the overall models of urbanicity and elevated CRP, men and women showed different patterns in the association between urbanicity components and inflammation by age (Figure 2). We saw three main patterns in the association of urbanicity components and inflammation in men in the unadjusted models, depicted in Figure 2 (solid lines). In general, younger men had a higher probability of inflammation with increasing urbanization while older men either showed declines in the probability of moderately elevated CRP with age (shown for housing, but also seen for population density, education, communication, health services, diversity, modern markets, and social services) or less steep positive increases in risk of inflammation with increasing urbanization (shown for economics, but also seen in sanitation and traditional markets). A third pattern, with no age interaction in the association between the component and inflammation, was found and plotted for the transportation component. In the age-adjusted multilevel logistic models (Table 3, Model 1a), improved housing infrastructure, greater access to traditional food and fuel markets, and greater community-level economic activity were associated with higher odds of moderate inflammation in younger men (aged 18-40).

In contrast, the probability of moderate inflammation tended to decrease with increasing urbanization component scores in younger women and increase in older women. We found three main patterns of association (Figure 2, solid lines) in women in the unadjusted models. As shown for the housing component, the probability of moderate inflammation for younger women was lower than older women and declined with increasing urbanization. A similar pattern was found for the education, communication, and diversity components. For several other components -- transportation (shown in Figure 2), density, sanitation, and traditional markets-younger women had a marginally higher probability of inflammation at the lowest level of urbanization, but nonetheless showed declines with increasing urbanization, in contrast to older women who had an increased probability of elevated CRP with increasing urbanization. In three components, economics (shown in Figure 2), modern markets, and 
social services, the probability of inflammation increased for younger women as well as older women. Health services (not shown) evidenced a different pattern than any other component, showing a positive association only in women over 60 . In age-adjusted multilevel logistic models (Table 3, Model 1b), the odds of inflammation were significantly higher with increasing transportation and diversity scores in younger women; with increasing sanitation, housing, communication, economics, diversity, and modern market scores in middle-aged women (40-60); and with increasing education, communication, traditional market, and economics scores in women over 60 .

\section{Do individual-level risk factors explain component-CRP association?-}

Controlling for individual-level risk factors (sociodemographic, obesogenic and pathogenic; Supplemental Table 1) that may mediate the association between community-level components of urbanization and individual inflammation had little attenuating effect on the associations between housing, traditional markets, or economic activity and moderately elevated CRP in younger men (Figure 2 dashed lines; Table 3, Model 2a). Each component remained a significant independent predictor of CRP.

The situation in women was more complicated (Figure 2 dashed lines). Controlling for sociodemographic risk factors (Supplemental Table 2) attenuated the effects of all components in younger women and communication in older women. Additionally controlling for obesogenic risk factors attenuated the effects of sanitation, communication, and diversity in women aged 40-60 and the effects of education, traditional markets, and economics in women over 60. Education, housing quality, economics and modern markets remained significant independent predictors of elevated CRP in the middle age group (4060 years) after controlling for all sociodemographic, obesogenic and pathogenic risk factors (Table 3, Model 2b).

\section{Discussion}

Like previous research looking at urban vs. rural differences in inflammation in China (Ye et al., 2007; Zhao et al., 2010) and other developing countries (McDade et al., 2008; Yajnik et al., 2008), we found that adults living in more urban communities were more likely to have moderate inflammation than those living in less developed communities. However, unlike much previous research that has focused on a single governmentally- or geographicallydefined dichotomous measure of urban/rural location, we used a multicomponent measure that reflects both the physical environments and also the social contexts that accompany urbanization (Jones-Smith and Popkin, 2010). This multidimensionality is particularly important in China given the rapidity and non-uniformity of urbanization (Gong et al., 2012; $\mathrm{Li}$ et al., 2012) and concerns that an urbanrural dichotomy is unlikely to capture the continuum of living conditions and health outcomes experienced by individuals living in different communities (Dahly and Adair, 2007; McDade and Adair, 2001; Van de Poel et al., 2009).

We found considerable heterogeneity in the association between community-level measures of urbanicity and its components and inflammation across sex and age strata in Chinese adults that was not completely explained by individual risk factors. Increasing levels of 
urbanicity, measured through the overall urbanicity index score or through its individual components, were independently associated with greater odds of elevated CRP in younger men but not in those aged 40 or older. Conversely, greater levels of urbanicity were associated with greater odds of elevated CRP in women over 40 but not among younger women. Women appeared to be more sensitive to the individual components of urbanization than men. Many of the components were associated with a lower probability of moderately elevated CRP in younger women, but a greater probability of inflammation in women 40 and older. These components remained independently associated with CRP in adjusted models in middle-aged women but not in younger (18-40 years) or older (>60 years) women. The attenuation of many of the components with the inclusion of education and/or obesogenic factors in younger and older women suggests that, for these age groups at least, increasing weight and waist circumferences accompanying urban lifestyles may underlie the association between urbanization and inflammation. On the other hand, the persistent influence of urbanicity components when controlling for individual-level sociodemographic, obesogenic, and pathogenic risk factors in younger men and women aged 40-60 suggests that elements of the community environment may directly or indirectly place individuals at risk for inflammation.

Unlike much previous work on neighborhoods and health that has used aggregated individual-level data, such as average household income, as a proxy for social and physical environmental characteristics (Gallo et al., 2012; Holmes and Marcelli, 2012; Petersen et al., 2008), we examined these social and physical characteristics of communities directly. We hypothesized that three main pathways -obesogenic, pathogenic, and psychosocial stresswould link the social and physical environments captured in our urbanization components to the process of inflammation in Chinese adults. More specifically, we hypothesized that some components of urbanization, such as greater access to markets and improved transportation infrastructure, would be associated with greater risk of inflammation, since these environmental changes are associated with the consumption of high-fat, high-energy Western diets, reduced physical activity, and subsequent visceral adiposity, a main source of pro-inflammatory cytokines. Conversely, we hypothesized that others, such as higher economic development, improved housing quality or availability of health services, would be associated with lower risk of inflammation since individuals would be exposed to fewer of the environmental pathogens that activate the inflammatory response and/or receive increased treatment for infectious illnesses or underlying pro-inflammatory conditions like diabetes or hypertension. Finally, we hypothesized that increasing population density and income diversity would serve as markers of pyschosocial stress and would increase the risk of inflammation through activation of the stress response.

While we found that a number of components were significantly associated with inflammation -- including housing conditions, sanitation, community economic and educational levels, transportation and communication infrastructure, and access to traditional and modern markets, the direction of these associations differed from our theoretical expectations and previous empirical research in the United States and other Western countries. Unlike a number of previous studies documenting significant inverse associations between CRP and individual and community income and education level (Gallo et al., 2012; Nazmi and Victora, 2007; Petersen et al., 2008; Pollitt et al., 2007), we found that 
community-level education, income, and housing quality were all associated with greater odds of moderate inflammation in younger Chinese men and Chinese women over 40. Further, we found no ameliorating effects of community-level health or social service availability on individual-level risk of inflammation, despite previous hypotheses that access to these services may underlie the inverse relationship between state- and census tract-level income and CRP levels (Clark et al., 2012; Holmes and Marcelli, 2012).

A number of components previously linked to inflammation in urbanization and neighborhood health research, including sanitation (McDade et al., 2008; Williams and McDade, 2009), income diversity (Gallo et al., 2012; Nazmi and Victora, 2007; Petersen et al., 2008), and education (Nazmi and Victora, 2007) were attenuated in the women in our study when we controlled for individual-level sociodemographic and obesogenic risk factors. Further controlling for pathogen burden had no additional attenuating effect. These results suggest that some of the positive association we found between higher urban component scores and inflammation is due to the high waist circumference, overweight, and/or high fat diets that accompany urbanization. Changes in diet, physical activity, and smoking have been proposed to underlie some of the associations between neighborhoods and health outcomes (Diez Roux and Mair, 2010), and each has a direct and indirect, through increased visceral adiposity, association with inflammation (Kushner et al., 2006). These characteristics also differed with urbanicity in our study. Obesity, physical inactivity and high intake of dietary fat remain more prevalent in towns and cities (versus rural areas) in China, though they are becoming more common in rural areas with economic growth (Gong et al., 2012; Popkin et al., 2012; Popkin and Du, 2003; Popkin et al., 2002).

A number of these associations between community-level urbanization and inflammation persisted in the younger men and middle-aged women in our sample, however, even when controlling for individual-level factors. That these community-level measures of education, housing conditions, economics, and access to markets were significantly associated with greater odds of inflammation after controlling for sociodemographic, obesogenic, and pathogenic factors provides evidence that unmeasured facets of the urbanizing environment, such as psychosocial and environmental stressors, remain important. In the United States, residence in lower socioeconomic neighborhoods increases exposure to chronic stressors, such as pollution, crowding, and social disorder (Cagney and Cornwell, 2010; Gallo et al., 2012; Holmes and Marcelli, 2012). Such exposures may be pro-inflammatory, activating the immune system, the hypothalamic-adrenal axis and sympathetic nervous system stress responses (Evans and Kantrowitz, 2002; Segerstrom and Miller, 2004). Similar psychosocial and environmental challenges are likely to accompany urban living in China and may underlie the positive relationships we saw between urbanization components and inflammation. Unfortunately, measures of neighborhood quality and psychosocial stress were not collected in the 2009 wave of the CHNS, so we are unable to test these pathways directly. Further research is needed to examine whether the association between urban components and inflammation is mediated through individual perceptions of stress, neighborhood quality, and/or social cohesion in China.

Urbanization in China has been characterized by pollution, social isolation, overcrowding, changes in diet and physical activity, and inadequate infrastructures for providing water, 
waste and sanitation services (Gong et al., 2012; Li et al., 2012; Popkin and Du, 2003; Wen et al., 2010). While each of the component scores increased across tertiles of overall urbanicity so that housing quality, for example, remained better at higher levels of overall urbanicity, longitudinal assessment of change in urbanicity components in the CHNS has shown that not all components have improved over time in all communities. In some communities at both lower and higher levels of urbanicity, losses were seen in health infrastructure, access to modern and traditional markets, and transportation (Jones-Smith and Popkin 2010). Reduced access to services and markets, increased population density, and lower social cohesion may contribute to psychosocial stress in China (Wen et al., 2010), thus increasing the risk of inflammation through immune and sympathetic nervous system pathways. Self-rated health status, a measure of health-related quality of life, also differs with urbanization in China. Adults rate their health best in mid-sized Chinese cities compared to rural areas, smaller cities, and large cities, a finding attributed to the lower stress-levels, better housing, and better environmental quality seen in middle-sized cities (Sun et al., 2011).

Along with this heterogeneity in the association between urbanization components and inflammation, we found significant sex- and age-differences in the associations between urbanization and inflammation. Overall, more significant associations were found between urbanization components and inflammation in women than men, which could reflect sexbased differences in the work and home environments and lifestyles of men and women in China (Fan, 2003) that place them at risk for inflammation. Previous research has documented differences in overweight and the prevalence of the metabolic syndrome (METs) in Chinese men and women by urban and rural residence, with urban men being at greater risk of overweight and METs than urban women and the reverse in rural areas (Weng et al., 2007). In our sample, patterns of overweight and high waist circumference with urbanicity also vary by sex and age strata (data not shown). Men aged 18-40 years have a significantly higher prevalence of overweight than younger women, particularly in highly urban areas. Conversely, older women (>40 years) have a significantly higher prevalence of high waist circumference than older men at all levels of urbanicity. These sexand age-based differences in pattering suggest that men and women are differentially exposed to the components of urbanization during their life course; for example, younger men may be more likely than older men to eat Western diets and have jobs that do not require physical labor while middle-aged and older women may be less likely than younger women to participate in leisure-time physical activity (Ng et al., 2009; Popkin 1999).

Age-related differences in exposure or sensitivity to residential neighborhoods may also account for some of the differences we see in younger vs. older individuals. Previous research suggests that older adults both have greater exposure to residential neighborhood conditions, such as poor housing quality, exposure to environmental contaminants, and lack of social cohesion, as they retire and center their activities within the home and also have greater immune sensitivity to these aspects of neighborhood quality than younger adults (Cagney and Cornwell, 2010; Williams and McDade, 2009). Alternatively, the documented age patterns may stem from the cross-sectional nature of our analysis. The rapidity of urbanization in China means that the current environment is not necessarily the most salient for older individuals. Research in other developing contexts suggests that early life 
exposures may be important in shaping long-term patterns of inflammation (McDade et al., 2009; Nazmi et al., 2010b). Longitudinal examination of exposure to changing urban environments and the trajectory of inflammation across the life course is needed to better identify the specific characteristics of urban environments that promote inflammation.

Despite the strengths of the multicomponent urbanicity index and our ability to examine social and physical aspects of urbanization at the community-level in an important context, this study has several limitations. First, biomarkers were first collected in the 2009 wave of the CHNS and, consequently, we are limited to a CRP measurement at a single time point. Although the majority of studies on inflammation rely on a single measure of CRP as a marker of chronic inflammation, a single measure cannot rule out that an elevated CRP value represents recovery from a recent illness rather than chronic elevations in baseline inflammation. Serial measures are necessary to distinguish between these possibilities; however, we limited our sample to those with CRP levels below $10 \mathrm{mg} / \mathrm{L}$, a commonly accepted marker of acute illness, and controlled for the presence of any illness symptoms in the preceding month in the adjusted models to mitigate the error introduced by the single CRP measurement. Second, like other multilevel studies supporting a relationship between place and health, we are limited in our ability to make causal interpretations by our crosssectional analysis (Daniel et al., 2008). Social and physical environments exert numerous issues with confounding and endogeneity that cannot be addressed in cross-sectional analysis (Daniel et al., 2008; Diez Roux and Mair, 2010). Third, and most important, we do not have direct measures of environmental quality, such as measures of environmental fecal contamination, water or air quality, or presence of pesticides, arsenic and other toxic chemicals in the air, water or food sources, which characterize unhealthy Chinese environments (Chen et al., 2013). While we are not able to measure these environmental contaminants directly, a number of the components of the urbanicity scale, including the housing quality, transportation infrastructure and sanitation components, were designed to capture pollution indirectly. Our analysis represents an important first step in establishing the contemporaneous associations between components of urbanization, individual risk factors and inflammation that may help elucidate the environmental and biological pathways linking place to inflammation in a developing country context.

\section{Conclusion}

In summary, we documented significant positive relationships between overall urbanicity and inflammation in younger men and women aged 40-60. Components of the urbanizing environment, including education, housing quality, access to markets, and community-level socioeconomic status, had persistent effects on inflammation that differed by age and sex even when controlling for a host of individual risk factors that have been proposed to underlie the association between urbanization and chronic disease risk. These persistent effects of local environments highlight the important role of place of residence on health outcomes in a country undergoing rapid urbanization. Further, the age and sex differences seen in the associations between physical and social components of the community and risk of inflammation underscore the importance of understanding the effect of urban environments in shaping individual vulnerability to poor health outcomes and for designing community-level interventions. While further longitudinal research is needed to understand 
the effect of exposure to changing urban environments on the trajectory of inflammation across the life course, these findings provide preliminary evidence that urbanicity is associated with inflammation risk in even young adults. Our results suggest that inflammation may become an increasingly important public health concern with continued urbanization and environmental change in China and elsewhere in the developing world.

\section{Supplementary Material}

Refer to Web version on PubMed Central for supplementary material.

\section{Acknowledgments}

This work was supported by NIH NICHD (K01 HD071948-01). KMH was supported by the CPC Population Research Training grant (T32 HD007168). PGL acknowledges support from NHLBI (R01HL108427). We thank the Institute of Nutrition and Food Safety, China Center for Disease Control and Prevention, the Carolina Population Center, the University of North Carolina at Chapel Hill, the NIH (R01-HD30880, DK056350, 5 R24 HD050924, and R01-HD38700) and the Fogarty International Center, NIH for financial support for the CHNS data collection and analysis files from 1989 to 2011 and future surveys, and the China-Japan Friendship Hospital, Ministry of Health for support for CHNS 2009. We thank the Carolina Population Center at the University of North Carolina at Chapel Hill (5 R24 HD050924) for general support.

\section{References}

Ainsworth BE, Haskell WL, Whitt MC, Irwin ML, Swartz AM, Strath SJ, O'Brien WL, Bassett DR Jr. Schmitz KH, Emplaincourt PO, Jacobs DR Jr. Leon AS. Compendium of physical activities: an update of activity codes and MET intensities. Medicine and science in sports and exercise. 2000; 32:S498-504. [PubMed: 10993420]

Alberti K, Zimmet P, Shaw J. Metabolic syndrome, A new worldwide definition. A Consensus Statement from the International Diabetes Federation. Diabetic Medicine. 2006; 23:469-480. [PubMed: 16681555]

Browning CR, Cagney KA, Iveniuk J. Neighborhood stressors and cardiovascular health: Crime and C-reactive protein in Dallas, USA. Social Science \& Medicine. 2012; 75:1271-1279. [PubMed: 22766311]

Cagney KA, Cornwell EY. Neighborhoods and Health in Later Life: The Intersection of Biology and Community. Annual Review of Gerontology and Geriatrics. 2010; 30:323-348.

Chen Y, Ebenstein A, Greenstone M, Li H. Evidence on the impact of sustained exposure to air pollution on life expectancy from China's Huai River policy. Proceedings of the National Academy of Sciences. 2013; 110:12936-12941.

Clark CR, Ridker PM, Ommerborn MJ, Huisingh CE, Coull B, Buring JE, Berkman LF. Cardiovascular inflammation in healthy women multilevel associations with state-level prosperity, productivity and income inequality. BMC Public Health. 2012; 12:211. [PubMed: 22433166]

Dahly DL, Adair LS. Quantifying the urban environment: a scale measure of urbanicity outperforms the urban-rural dichotomy. Social Science \& Medicine. 2007; 64:1407-1419. [PubMed: 17196724]

Daniel M, Moore S, Kestens Y. Framing the biosocial pathways underlying associations between place adn cardiometabolic disease. Health \& Place. 2008; 14:117-132. [PubMed: 17590377]

Diez Roux AV, Mair C. Neighborhoods and health. Annals of the New York Academy of Sciences. 2010; 1186:125-145. [PubMed: 20201871]

Evans GW, Kantrowitz E. Socioeconomic status and health: the potential role of environmental risk exposure. Annual review of public health. 2002; 23:303-331.

Fan CC. Rural-urban migration and gender division of labor in transitional China. International Journal of Urban and Regional Research. 2003; 27:24-47.

Gallo LC, Fortmann AL, de los Monteros KE, Mills PJ, Barrett-Connor E, Roesch SC, Matthews KA. Individual and Neighborhood Socioeconomic Status and Inflammation in Mexican American 
Women: What is the Role of Obesity? Psychosomatic Medicine. 2012; 74:535-542. [PubMed: 22582313]

Gong P, Liang S, Carlton EJ, Jiang Q, Wu J, Wang L, Remais JV. Urbanisation and health in China. Lancet. 2012; 379:843-852. [PubMed: 22386037]

Hansel A, Hong S, Camara RJ, Von Kaenel R. Inflammation as a psychophysiological biomarker in chronic psychosocial stress. Neuroscience \& Biobehavioral Reviews. 2010; 35:115-121. [PubMed: 20026349]

Holmes LM, Marcelli EA. Neighborhoods and systematic inflammation: High CRP among legal and unauthorized Brazillian migrants. Health \& Place. 2012; 18:683-693. [PubMed: 22401803]

Jones-Smith JC, Popkin BM. Understanding community context and adult health changes in China: development of an urbanicity scale. Social Science \& Medicine. 2010; 71:1436-1446. [PubMed: 20810197]

Kushner I, Rzewnicki D, Samols D. What does minor elevation of C-reactive protein signify? The American journal of medicine. 2006; 119:166-e17. [PubMed: 16443421]

Li X, Wang C, Zhang G, Xiao L, Dixon J. Urbanisation and human health in China: spatial features and a systemic perspective. Environmental Science and Pollution Research. 2012; 19:1375-1384. [PubMed: 22743987]

McDade TW, Adair LS. Defining the "urban" in urbanization and health: a factor analysis approach. Social Science \& Medicine. 2001; 53:55-70. [PubMed: 11380161]

McDade TW, Rutherford J, Adair L, Kuzawa CW. Early origins of inflammation: microbial exposures in infancy predict lower levels of C-reactive protein in adulthood. Proceedings of the Royal Society B: Biological Sciences. 2009; 277:1129-1137.

McDade TW, Rutherford JN, Adair L, Kuzawa C. Adiposity and pathogen exposure predict C-reactive protein in Filipino women. Journal of Nutrition. 2008; 138:2442-2447. [PubMed: 19022970]

Nazmi A, Diez Roux AV, Ranjit N, Seeman TE, Jenny NS. Cross-sectional and longitudinal associations of neighborhood characteristics with inflammatory markers: Findings from the multiethnic study of atherosclerosis. Health \& Place. 2010a; 16:1104-1112. [PubMed: 20667763]

Nazmi A, Oliveira IO, Horta BL, Gigante DP, Victora CG. Lifecourse socioeconomic trajectories and C-reactive protein levels in young adults: findings from a Brazilian birth cohort. Social Science \& Medicine. 2010b; 70:1229-1236. [PubMed: 20137842]

Nazmi A, Victora CG. Socioeconomic and racial/ethnic differentials of C-reactive protein levels: a systematic review of population-based studies. BMC Public Health. 2007; 7:212. [PubMed: 17705867]

Ng SW, Norton EC, Popkin BM. Why have physical activity levels declined among Chinese adults? Findings from the 1991-2006 China Health and Nutrition Surveys. Social Science \& Medicine. 2009; 68:1305-1314. [PubMed: 19232811]

Pearson TA, Mensah GA, Hong Y, Smith SC Jr. CDC/AHA Workshop on Markers of Inflammation and Cardiovascular Disease: Application to Clinical and Public Health Practice: overview. Circulation. 2004; 110:e543-544. [PubMed: 15611378]

Petersen KL, Marsland AL, FLory J, Vortruba-Drzal E, Muldoon MF, Manuck SB. Community Socioeconomic Status is Associated with Circulating Interleukin and C-Reactive Protein. Psychosomatic Medicine. 2008; 70:646-652. [PubMed: 18606725]

Pollitt RA, Kaufman JS, Rose KM, Diez-Roux AV, Zeng D, Heiss G. Early-life and adult socioeconomic status and inflammatory risk markers in adulthood. European Journal of Epidemiology. 2007; 22:55-66. [PubMed: 17225957]

Popkin BM. Urbanization, lifestyle changes and the nutrition transition. World Development. 1999; 27:1905-1916.

Popkin BM, Adair LS, Ng SW. Global nutrition transition and the pandemic of obesity in developing countries. Nutrition reviews. 2012; 70:3-21. [PubMed: 22221213]

Popkin BM, Du S. Dynamics of the nutrition transition toward the animal foods sector in China and its implications: a worried perspective. The Journal of Nutrition. 2003; 133:3898S-3906S. [PubMed: 14672288] 
Popkin BM, Du S, Zhai F, Zhang B. Cohort Profile: The China Health and Nutrition Survey-monitoring and understanding socio-economic and health change in China, 1989-2011. International Journal of Epidemiology. 2010; 39:1435-1440. [PubMed: 19887509]

Popkin BM, Lu B, Zhai F. Understanding the nutrition transition: measuring rapid dietary changes in transitional countries. Public Health Nutrition. 2002; 5:947-953. [PubMed: 12633520]

Pradhan AD, Manson JE, Rifai N, Buring JE, Ridker PM. C-reactive protein, interleukin 6, and risk of developing type 2 diabetes mellitus. JAMA. 2001; 286:327-334. [PubMed: 11466099]

Segerstrom SC, Miller GE. Psychological stress and the human immune system: a meta-analytic study of 30 years of inquiry. Psychological bulletin. 2004; 130:601-630. [PubMed: 15250815]

Sun S, Chen J, Johannesson M, Kind P, Xu L, Zhang Y, Burstrom K. Regional differences in health status in China: Population health-realted quality of life results from the National Health Services Survey 2008. Health \& Place. 2011; 17:671-680. [PubMed: 21334961]

Van de Poel E, O'Donnell O, Van Doorslaer E. Urbanization and the spread of diseases of affluence in China. Economics \& Human Biology. 2009; 7:200-216. [PubMed: 19560989]

Vlahov D, Galea S. Urbanization, urbanicity, and health. Journal of Urban Health. 2002; 79:S1-S12. [PubMed: 12473694]

Vlahov D, Galea S, Freudenberg N. The Urban Health “Advantage”. Journal of Urban Health. 2005; 82:1-4. [PubMed: 15738341]

Wang L. Summary Report of China Nutrition and Health Survey 2002. Beijing: People's Medical Publishing House. 2002

Wen M, Fan J, Jin L, Wang G. Neighborhood effects on health on migrants and natives in Shanghai, China. Health \& Place. 2010; 16:452-460. [PubMed: 20060767]

Weng X, Liu Y, Ma J, Wang W, Yang G, Caballero B. An urban-rural comparison of the prevalence of the metabolic syndrome in Eastern China. Public health nutrition. 2007; 10:131-136. [PubMed: 17261221]

Williams SR, McDade TW. The use of dried blood spot sampling in the national social life, health, and aging project. The journals of gerontology. Series B, Psychological sciences and social sciences. 2009; 64:i131-136.

Working group on Obesity in China. Department of Disease Control and Ministry of Health. Biomedical \& Environmental Science. 2004. The guidelines for prevention and control of overweight and obesity in Chinese adults; p. 1-35.

Yajnik C, Joglekar C, Lubree H, Rege S, Naik S, Bhat D, Uradey B, Raut K, Shetty P, Yudkin J. Adiposity, inflammation and hyperglycaemia in rural and urban Indian men: Coronary Risk of Insulin Sensitivity in Indian Subjects (CRISIS) Study. Diabetologia. 2008; 51:39-46. [PubMed: 17972060]

Ye X, Yu Z, Li H, Franco OH, Liu Y, Lin X. Distributions of C-reactive protein and its association with metabolic syndrome in middle-aged and older Chinese people. Journal of the American College of Cardiology. 2007; 49:1798-1805. [PubMed: 17466231]

Zhao Y, Wang R, Ma X, Yan X, Zhang Z, He X, He J. Distribution of C-reactive protein and its association with cardiovascular risk factors in a population-based sample of Chinese. Disease markers. 2010; 28:333-342. [PubMed: 20683147] 


\section{Research Highlights}

- Higher levels of urbanicity are associated with greater risk of inflammation

- Physical and social components of urbanization are associated with elevated CRP

- Sensitivity of inflammation to urbanization differs by age and sex

- Individual risk factors do not completely explain the effect of urbanization on CRP 

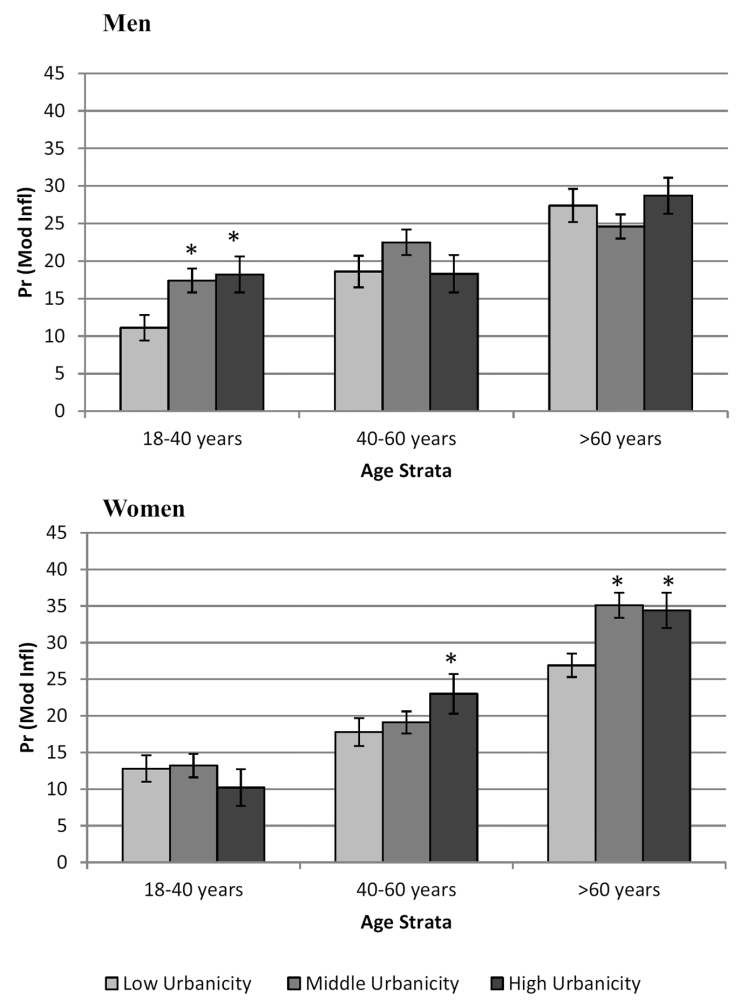

Figure 1. Predicted probability of elevated CRP $(3-10 \mathrm{mg} / \mathrm{L})$ across tertiles of urbanization by age strata and sex

*significant urbanicity effect within age strata, $\mathrm{p}<0.05$. 

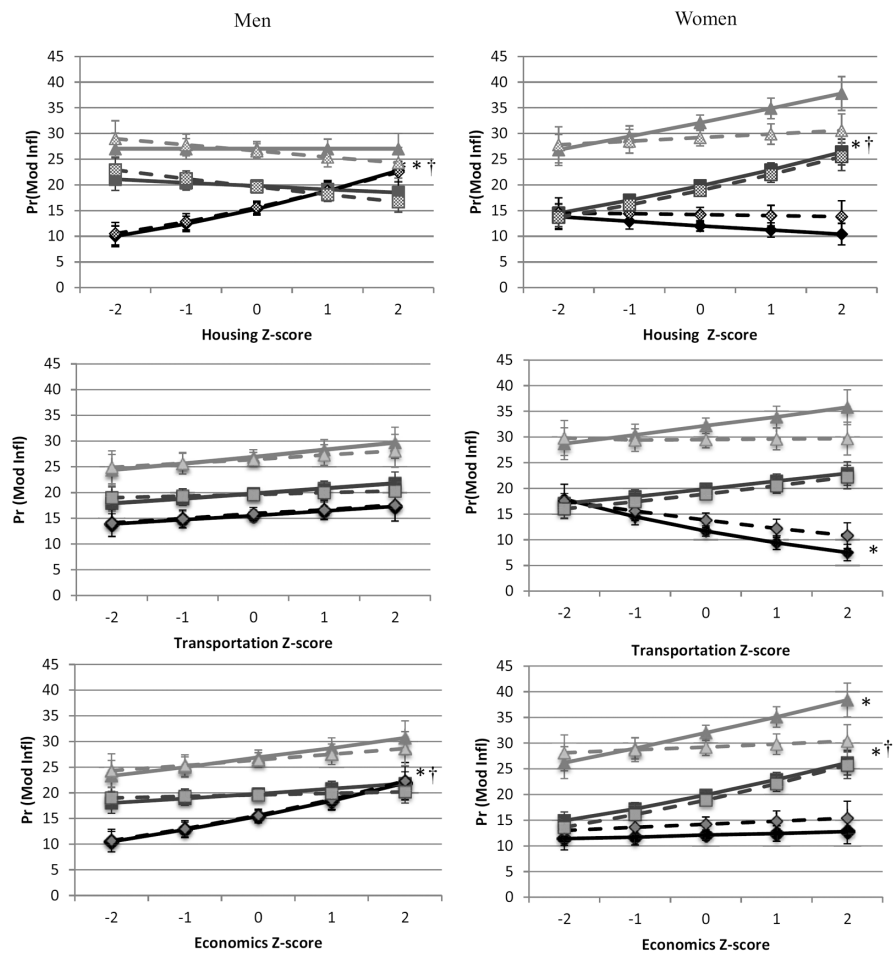

Figure 2. Unadjusted and adjusted sex- and age-strata differences in the predicted probabilities of moderately elevated CRP with higher urbanization component scores

*significant slope (in age strata) in multilevel logistic model, controlling for clustering by community, $\mathrm{p}<0.05$. $\dagger$ significant slope (in age strata) in adjusted model controlling for overweight, high waist circumference, high fat diet, physical activity, smoking, income, education, infectious illness symptoms, region and clustering by community, $\mathrm{p}<0.05$. 


\section{Table 1}

\section{Description of Urbanization Components ${ }^{a}$}

\begin{tabular}{ll}
\hline Components & Description \\
\hline Population Density & Population per km² \\
Education & Average highest attained level for adults \\
Sanitation & Availability of treated water and presence of excrement in public space \\
Housing & Availability of electricity, water, gas \\
Transportation & Road types and availability of transit services \\
Communication & Media availability in the community and percent of households with electronics \\
Health Infrastructure & Type, distance and number of health services (clinics, hospitals, pharmacies, etc) in the community \\
Traditional Markets & Types, distances and business hours of food and fuel markets \\
Economic Activity & Daily wage for average male worker and \% community engaged in nonagricultural labor \\
Diversity & Community variance in education and income levels \\
Modern Markets & Quantity of supermarkets and modern eating establishments \\
Social Services & Availability of insurance and child care centers \\
\hline
\end{tabular}

${ }^{a}$ Described in \$ \$Jones-Smith and Popkin, 2010\$\$. Points were assigned based on the characteristics listed above with higher scores indicating greater urbanization. 


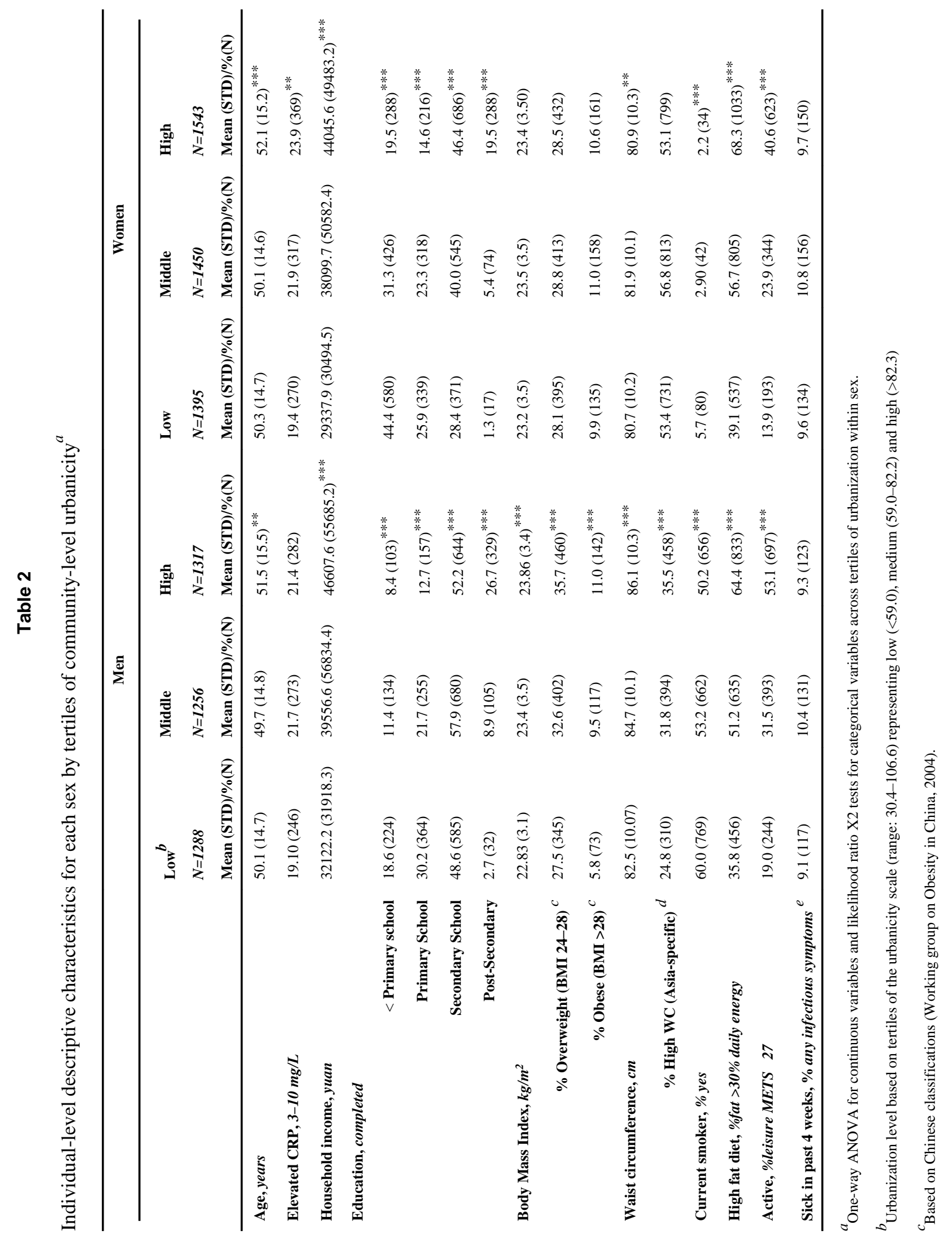




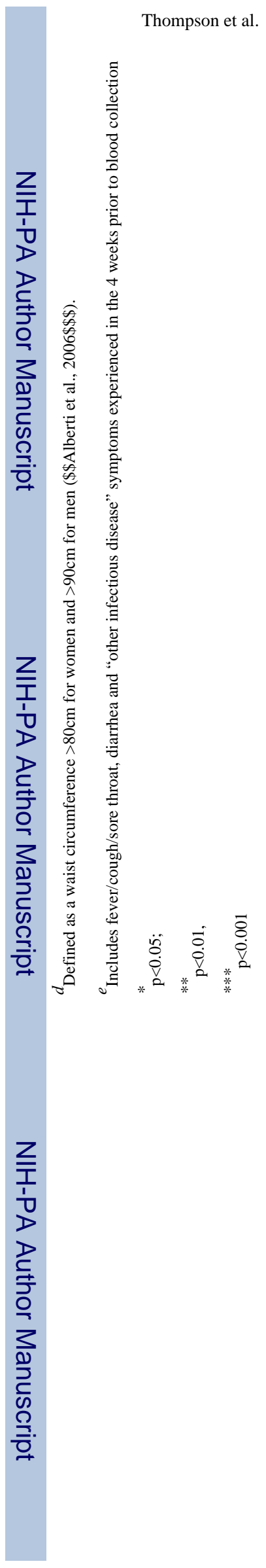

Page 21

Health Place. Author manuscript; available in PMC 2015 July 01. 
Table 3

Urbanization components and odds of elevated CRP

\begin{tabular}{|c|c|c|c|c|c|c|c|c|}
\hline \multirow{4}{*}{$\begin{array}{l}\text { Urbanization } \\
\text { components by age } \\
\text { strata } \\
\text { Population Density 18-40 } \\
\text { years }\end{array}$} & \multicolumn{4}{|c|}{ Men } & \multicolumn{4}{|c|}{ Women } \\
\hline & \multicolumn{2}{|c|}{$\begin{array}{c}\text { Model 1a: Age and } \\
\quad \text { Residence }{ }^{a}\end{array}$} & \multicolumn{2}{|c|}{$\begin{array}{c}\text { Model 2a: Individual } \\
\quad \text { Risk Factors } b\end{array}$} & \multicolumn{2}{|c|}{$\begin{array}{c}\text { Model 1b: Age and } \\
\text { Residence }^{a}\end{array}$} & \multicolumn{2}{|c|}{$\begin{array}{c}\text { Model 2b: Individual } \\
\quad \text { Risk Factors }\end{array}$} \\
\hline & \multicolumn{2}{|c|}{$\mathrm{OR}^{c}(95 \% \mathrm{CI})$} & \multicolumn{2}{|c|}{ OR $(95 \% \mathrm{CI})$} & \multicolumn{2}{|c|}{ OR $(95 \% \mathrm{CI})$} & \multicolumn{2}{|c|}{ OR $(95 \% \mathrm{CI})$} \\
\hline & 1.03 & {$[0.87,1.23]$} & 1.12 & {$[0.91,1.36]$} & 0.94 & {$[0.78,1.13]$} & 0.96 & {$[0.78,1.19]$} \\
\hline $40-60$ years & 0.96 & {$[0.85,1.08]$} & 0.89 & {$[0.78,1.02]$} & 1.04 & {$[0.93,1.17]$} & 1.06 & {$[0.94,1.21]$} \\
\hline $60+$ years & 1.00 & {$[0.87,1.14]$} & 1 & {$[0.86,1.17]$} & 1.08 & {$[0.95,1.22]$} & 0.95 & {$[0.82,1.10]$} \\
\hline Education $18-40$ years & 1.06 & {$[0.88,1.28]$} & 1.1 & {$[0.88,1.36]$} & 0.82 & {$[0.66,1.03]$} & 0.94 & {$[0.73,1.21]$} \\
\hline $40-60$ years & 0.99 & {$[0.88,1.11]$} & 0.95 & {$[0.83,1.09]$} & 1.11 & {$[0.99,1.23]$} & $1.20^{* *}$ & {$[1.05,1.36]$} \\
\hline $60+$ years & 0.94 & {$[0.82,1.08]$} & 0.91 & {$[0.77,1.08]$} & $1.15^{*}$ & {$[1.01,1.30]$} & 1.14 & {$[0.98,1.33]$} \\
\hline Sanitation $18-40$ years & 1.18 & {$[0.99,1.40]$} & 1.22 & {$[0.99,1.51]$} & 0.92 & {$[0.77,1.11]$} & 0.91 & {$[0.73,1.13]$} \\
\hline 40-60 years & 1.03 & {$[0.92,1.16]$} & 0.99 & {$[0.87,1.13]$} & $1.20^{* *}$ & {$[1.07,1.36]$} & 1.1 & {$[0.97,1.26]$} \\
\hline $60+$ years & 1.05 & {$[0.91,1.20]$} & 0.97 & {$[0.83,1.14]$} & 1.14 & {$[1.00,1.29]$} & 1.01 & {$[0.87,1.17]$} \\
\hline Housing $18-40$ years & $1.28^{* *}$ & {$[1.06,1.54]$} & $1.37^{* *}$ & {$[1.09,1.70]$} & 0.92 & {$[0.77,1.11]$} & 0.98 & {$[0.78,1.22]$} \\
\hline 40-60 years & 0.96 & {$[0.85,1.08]$} & 0.92 & {$[0.81,1.06]$} & $1.20^{* *}$ & {$[1.07,1.36]$} & $1.23^{* *}$ & {$[1.07,1.41]$} \\
\hline $60+$ years & 1.00 & {$[0.87,1.15]$} & 0.94 & {$[0.80,1.10]$} & 1.14 & {$[1.00,1.29]$} & 1.03 & {$[0.89,1.20]$} \\
\hline $\begin{array}{l}\text { Transportatio n } 18-40 \\
\text { years }\end{array}$ & 1.07 & {$[0.89,1.28]$} & 1.08 & {$[0.88,1.32]$} & $0.78^{*}$ & {$[0.65,0.94]$} & 0.86 & {$[0.69,1.08]$} \\
\hline 40-60 years & 1.06 & {$[0.95,1.19]$} & 1.03 & {$[0.91,1.17]$} & 1.1 & {$[0.98,1.23]$} & 1.11 & {$[0.98,1.26]$} \\
\hline $60+$ years & 1.07 & {$[0.93,1.23]$} & 1.07 & {$[0.92,1.25]$} & 1.08 & {$[0.95,1.24]$} & 1 & {$[0.87,1.16]$} \\
\hline $\begin{array}{l}\text { Communicati on } 18-40 \\
\text { years }\end{array}$ & 1.06 & {$[0.88,1.26]$} & 1.04 & {$[0.84,1.27]$} & 0.86 & {$[0.71,1.04]$} & 0.89 & {$[0.71,1.11]$} \\
\hline $40-60$ years & 0.95 & {$[0.84,1.08]$} & 0.9 & {$[0.79,1.03]$} & $1.19^{* *}$ & {$[1.05,1.34]$} & 1.14 & {$[1.00,1.30]$} \\
\hline $60+$ years & 0.97 & {$[0.84,1.11]$} & 0.93 & {$[0.80,1.08]$} & $1.14^{*}$ & {$[1.00,1.30]$} & 1.04 & {$[0.91,1.20]$} \\
\hline $\begin{array}{l}\text { Health Infrastructure } 18- \\
40 \text { years }\end{array}$ & 1.13 & {$[0.95,1.34]$} & 1.16 & {$[0.94,1.43]$} & 0.93 & {$[0.77,1.12]$} & 0.87 & {$[0.70,1.08]$} \\
\hline 40-60 years & 1.02 & {$[0.91,1.15]$} & 1.01 & {$[0.89,1.14]$} & 0.96 & {$[0.86,1.07]$} & 0.98 & {$[0.86,1.10]$} \\
\hline $60+$ years & 0.97 & {$[0.84,1.11]$} & 0.96 & {$[0.82,1.12]$} & 1.12 & {$[0.97,1.28]$} & 1.05 & {$[0.91,1.22]$} \\
\hline $\begin{array}{l}\text { Traditional Markets 18-40 } \\
\text { years }\end{array}$ & $1.19^{*}$ & {$[1.00,1.41]$} & $1.24^{*}$ & {$[1.01,1.52]$} & 0.88 & {$[0.74,1.06]$} & 0.93 & {$[0.75,1.15]$} \\
\hline 40-60 years & 0.97 & {$[0.86,1.10]$} & 0.94 & {$[0.83,1.08]$} & 1.09 & {$[0.97,1.22]$} & 1.13 & {$[0.99,1.28]$} \\
\hline $60+$ years & 1.14 & {$[0.99,1.31]$} & $1.18^{*}$ & {$[1.01,1.38]$} & $1.18^{*}$ & {$[1.04,1.36]$} & 1.15 & {$[0.99,1.33]$} \\
\hline $\begin{array}{l}\text { Economic Activity 18-40 } \\
\text { years }\end{array}$ & $1.24^{*}$ & {$[1.03,1.49]$} & $1.32^{*}$ & {$[1.07,1.64]$} & 1.03 & {$[0.85,1.25]$} & 1.04 & {$[0.83,1.31]$} \\
\hline $40-60$ years & 1.06 & {$[0.94,1.19]$} & 1.02 & {$[0.90,1.16]$} & $1.19^{* *}$ & {$[1.07,1.34]$} & $1.23^{* *}$ & {$[1.08,1.40]$} \\
\hline $60+$ years & 1.10 & {$[0.95,1.27]$} & 1.05 & {$[0.89,1.23]$} & $1.15^{*}$ & {$[1.01,1.31]$} & 1.03 & {$[0.89,1.19]$} \\
\hline Diversity $18-40$ years & 1.12 & {$[0.94,1.33]$} & 1.2 & {$[0.98,1.46]$} & $0.79^{*}$ & {$[0.64,0.97]$} & 0.84 & {$[0.67,1.07]$} \\
\hline $40-60$ years & 1.03 & {$[0.92,1.17]$} & 1 & {$[0.88,1.15]$} & $1.14^{*}$ & {$[1.02,1.28]$} & 1.13 & {$[0.99,1.28]$} \\
\hline $60+$ years & 0.98 & {$[0.86,1.12]$} & 0.96 & {$[0.83,1.12]$} & 1.09 & {$[0.96,1.23]$} & 0.96 & {$[0.83,1.10]$} \\
\hline
\end{tabular}




\begin{tabular}{llccccccc}
\hline & \multicolumn{1}{c}{ Men } & \multicolumn{4}{c}{ Women } \\
\hline $\begin{array}{l}\text { Modern Markets 18-40 } \\
\text { years }\end{array}$ & 1.18 & {$[0.99,1.40]$} & $1.23^{*}$ & {$[1.00,1.50]$} & 0.99 & {$[0.82,1.20]$} & 1.04 & {$[0.83,1.29]$} \\
40-60 years & 0.98 & {$[0.87,1.10]$} & 0.93 & {$[0.82,1.06]$} & $1.20^{* *}$ & {$[1.07,1.35]$} & $1.23^{* *}$ & {$[1.08,1.40]$} \\
$\begin{array}{l}\text { 60+ years } \\
\text { Social Services 18-40 }\end{array}$ & 1.00 & {$[0.86,1.15]$} & 0.95 & {$[0.81,1.11]$} & 1.1 & {$[0.97,1.25]$} & 1.04 & {$[0.90,1.20]$} \\
years & 1.06 & {$[0.89,1.26]$} & 1.16 & {$[0.95,1.41]$} & 1.03 & {$[0.85,1.26]$} & 1.1 & {$[0.88,1.38]$} \\
$40-60$ years & 0.96 & {$[0.86,1.08]$} & 0.93 & {$[0.82,1.06]$} & 0.99 & {$[0.88,1.11]$} & 0.99 & {$[0.87,1.12]$} \\
60+ years & 0.95 & {$[0.83,1.09]$} & 0.9 & {$[0.77,1.05]$} & 1.05 & {$[0.93,1.19]$} & 0.98 & {$[0.85,1.12]$} \\
\hline
\end{tabular}

${ }^{a}$ Models specified to provide a slope for each age category and control for clustering by community.

${ }^{b}$ Models specified to provide a slope for each age category and control for overweight, high waist circumference, high fat diet, activity, smoking, income, education, infectious illness symptoms, region, and clustering by community.

${ }^{c}$ OR represent the effect of a 1-standard deviation increase in the component score for each age strata

* $\mathrm{p}<0.05$;

** $\mathrm{p}<0.01$ 